\title{
Single and vertically coupled type II quantum dots in a perpendicular magnetic field: exciton groundstate properties
}

\author{
K. L. Janssenst, B. Partoens \\ Departement Natuurkunde, Universiteit Antwerpen (UIA), Universiteitsplein 1, \\ B-2610 Antwerpen, Belgium
}

(November 4, 2018)

\begin{abstract}
The properties of an exciton in a type II quantum dot are studied under the influence of a perpendicular applied magnetic field. The dot is modelled by a quantum disk with radius $R$, thickness $d$ and the electron is confined in the disk, whereas the hole is located in the barrier. The exciton energy and wavefunctions are calculated using a Hartree-Fock mesh method. We distinguish two different regimes, namely $d<<2 R$ (the hole is located at the radial boundary of the disk) and $d>>2 R$ (the hole is located above and below the disk), for which angular momentum ( $l$ ) transitions are predicted with increasing magnetic field. We also considered a system of two vertically coupled dots where now an extra parameter is introduced, namely the interdot distance $d_{z}$. For each $l_{h}$ and for a sufficient large magnetic field, the ground state becomes spontaneous symmetry broken in which the electron and the hole move towards one of the dots. This transition is induced by the Coulomb interaction and leads to a magnetic field induced dipole moment. No such symmetry broken ground states are found for a single dot (and for three vertically coupled symmetric quantum disks). For a system of two vertically coupled truncated cones, which is asymmetric from the start, we still find angular momentum transitions. For a symmetric system of three vertically coupled quantum disks, the system resembles for small $d_{z}$ the pillar-like regime of a single dot, where the hole tends to stay at the radial boundary, which induces angular momentum transitions with increasing magnetic field. For larger $d_{z}$ the hole can sit between the disks and the $l_{h}=0$ state remains the groundstate for the whole $B$-region.
\end{abstract}

PACS: 73.21.La, 71.35.Ji, 85.35.Be

\section{INTRODUCTION}

The study of self-assembled quantum dots, as realized by the Stranski-Krastanow growth mode, has been a fascinating research area during the last decade. The possibility to discover new physics in these zero-dimensional (0D) structures together with possible applications in opto-electronics has led to many experimental and theoretical results. Most studies were devoted to type I quantum dots [2.9], where both electron and hole are spatially located inside the quantum dots. Relatively few works however were done for type II quantum dots, where electrons and holes are spatially separated. One expects from this type of dots interesting properties, such as large tunability of the emission energy and radiative lifetimes which are considerably longer than for their type I counterparts 12].

Within the group of type II quantum dots, one can still distinguish between two systems. Namely, the system which confines the electrons inside the quantum dot, and forms an antidot for the hole, like e.g. the InP/GaInP dots (if strain is neglected). Besides this, there are the systems, such as GaSb/GaAs [10] and InAs/Si [1], where the holes are confined within the dot, but where the electrons are located outside. Experimental studies on this type of quantum dots have been performed in Refs. [13 15] for the InP/GaInP system and in Refs. 16, 17] for the $\mathrm{GaSb} / \mathrm{GaAs}$ system. Theoretical investigations on $\operatorname{InP} / \mathrm{GaInP}$ type II dots were performed by Pryor et al. 18] and Tadic et al. [19], who used a strain-dependent $\mathbf{k} \cdot \mathbf{p}$ Hamiltonian to calculate the electronic structure. Nomura et al. 20] calculated the Landau levels in a high magnetic field, solving the Hartree equations self-consistently, while the present authors studied an exciton in a planar quantum dot using a Hartree-Fock approximation [21]. Lelong et al. 22] calculated the binding energy of excitons, charged excitons and biexcitons in $\mathrm{GaSb} / \mathrm{GaAs}$ dots using the Hartree-Fock approximation. The magneto-exciton in a $G a S b / G a A s$ dot was investigated by Kalameitsev et al. [23].

In the present paper we study a single electron-hole pair bound by the Coulomb interaction, i.e. an exciton, in a model type II quantum dot. The quantum dot is modelled by a quantum disk of finite height, which is an extension of our previous work [21] where we did not take into account the $z$-direction. As an example, we consider the electron in the dot and the hole in the barrier material. The conclusions of our paper are also valid for the reverse situation where the hole is inside the dot and the electron is outside the dot. In the present work, we neglect strain effects and for the confinement potential we use a hard wall of finite height, confining the electron inside the dot and repelling the hole to the barrier region. Furthermore, a magnetic field was applied in the growth direction, i.e. $\mathbf{B}=B \mathbf{e}_{z}$. 
The first part of this paper deals with the study of the exciton in a single dot. Investigation of the influence of the disk parameters, namely the radius $R$ and thickness $d$, showed that one can distinguish between two regimes: the disk-like regime and the pillar-like regime. For the first one, the disk thickness $d$ is much smaller than its diameter, i.e. $d<<2 R$, whereas the latter one describes the system with $d>>2 R$. Although the magnetic field couples only with the in-plane electron and hole coordinates, we will show that these systems, with different $z$-extension, behave very differently under an applied magnetic field.

In the second part of the paper, we study the properties of vertically coupled quantum dots. The interest of this study lies in the following. It is generally known that in reality self-assembled quantum dots resemble more our disk-like system than our pillar-like system. However, it is also known that it is not too difficult to form vertical stacks of these disk-like dots and thereby create a system which could behave like a pillar-like system.

The paper is organized as follows. In Sec. II, we give a brief discussion of our theoretical model. The numerical results in the absence of a magnetic field are presented in Sec. III. Section IV deals with the result for a perpendicular applied magnetic field. Part A of this Section discusses the results for the single dot disk-like system, whereas Part $\mathrm{B}$ is dedicated to the pillar-like system. Parts $\mathrm{C}$ and $\mathrm{D}$ deal with two vertically coupled dots, respectively disks and truncated cones. Parts E and F deal with three vertically coupled dots, respectively for small and large interdot distances. Finally, we make a small revision of the results for the single disk in Part G. Our results are summarized in Sec. V. In the Appendix, we discuss in more detail the numerical approach we used for the calculation of the Hartree integral.

\section{THEORETICAL MODEL}

We extended our previous approach [21], which was valid for planar dots, and include the $z$-direction. The energies and wavefunctions are solved within the effective mass approximation (with $m_{e}$ and $m_{h}$ the effective electron and

hole masses, respectively, $r_{e, h}=\sqrt{x_{e, h}^{2}+y_{e, h}^{2}}, \omega_{c, e}=e B / m_{e}$ and $\left.\omega_{c, h}=e B / m_{h}\right)$ and the Hartree-Fock (HF) single particle equations can be written as

$$
\begin{array}{r}
{\left[-\frac{\hbar^{2}}{2 m_{e}} \frac{1}{r_{e}} \frac{\partial}{\partial r_{e}}\left(r_{e} \frac{\partial}{\partial r_{e}}\right)-\frac{\hbar^{2}}{2 m_{e}} \frac{\partial^{2}}{\partial^{2} z_{e}}+\frac{\hbar^{2}}{2 m_{e}} \frac{l_{e}^{2}}{r_{e}^{2}}+\frac{l_{e}}{2} \hbar \omega_{c, e}+\frac{1}{8} m_{e} \omega_{c, e}^{2} r_{e}^{2}\right.} \\
\left.+V_{e}\left(r_{e}, z_{e}\right)-\frac{e^{2}}{4 \pi \epsilon} \int \frac{\rho_{h}\left(r^{\prime}, z^{\prime}\right)}{\left|\mathbf{r}-\mathbf{r}^{\prime}\right|} d \mathbf{r}^{\prime}\right] \psi_{e}\left(r_{e}, z_{e}\right)=\epsilon_{e} \psi_{e}\left(r_{e}, z_{e}\right), \\
{\left[-\frac{\hbar^{2}}{2 m_{h}} \frac{1}{r_{h}} \frac{\partial}{\partial r_{h}}\left(r_{h} \frac{\partial}{\partial r_{h}}\right)-\frac{\hbar^{2}}{2 m_{h}} \frac{\partial^{2}}{\partial^{2} z_{h}}+\frac{\hbar^{2}}{2 m_{h}} \frac{l_{h}^{2}}{r_{h}^{2}}-\frac{l_{h}}{2} \hbar \omega_{c, h}+\frac{1}{8} m_{h} \omega_{c, h}^{2} r_{h}^{2}\right.} \\
\left.+V_{h}\left(r_{h}, z_{h}\right)-\frac{e^{2}}{4 \pi \epsilon} \int \frac{\rho_{e}\left(r^{\prime}, z^{\prime}\right)}{\left|\mathbf{r}-\mathbf{r}^{\prime}\right|} d \mathbf{r}^{\prime}\right] \psi_{h}\left(r_{h}, z_{h}\right)=\epsilon_{h} \psi_{h}\left(r_{h}, z_{h}\right),
\end{array}
$$

where we made use of the axial symmetry by taking $\Psi_{e}\left(r_{e}, \varphi_{e}, z_{e}\right)=e^{i l_{e} \varphi_{e}} \psi_{e}\left(r_{e}, z_{e}\right)$ and $\Psi_{h}\left(r_{h}, \varphi_{h}, z_{h}\right)=$ $e^{i l_{h} \varphi_{h}} \psi_{h}\left(r_{h}, z_{h}\right)$, and where the densities $\rho_{e}\left(r^{\prime}, z^{\prime}\right)$ and $\rho_{h}\left(r^{\prime}, z^{\prime}\right)$ are given by respectively $\left|\Psi_{e}\left(r_{e}, \varphi_{e}, z_{e}\right)\right|^{2}$ and $\left|\Psi_{h}\left(r_{h}, \varphi_{h}, z_{h}\right)\right|^{2}$. We made use of a finite difference scheme to solve the Hartree-Fock equations. More details about the implementation of this finite difference scheme can be found in Refs. [25,21]. Note that we consider only a single electron and single hole and therefore there are no exchange terms. The reason that these equations can still be called HF lies in the fact that the self-interaction is excluded. As confinement potentials we take hard walls of finite height:

$$
V_{e, h}\left(r_{e}, z_{e}, r_{h}, z_{h}\right)= \begin{cases}V_{e, h}, & r_{e, h}>R \text { and }\left|z_{e, h}\right|>d / 2, \\ 0, & \text { otherwise, }\end{cases}
$$

with $R$ the radius of the disk, and where we took $V_{e}$ positive and $V_{h}$ negative. Note that the only good quantum number is the total angular momentum in the $z$-direction, defined by $L=l_{e}+l_{h}$.

We solved the equations self-consistently using an iterative procedure. Since only the electron is confined in the absence of any Coulomb interaction, we start with the free electron solution. The Hartree integrals are then integrated numerically as

$$
\int \frac{\rho\left(r^{\prime}, z^{\prime}\right)}{\left|\mathbf{r}-\mathbf{r}^{\prime}\right|} d \mathbf{r}^{\prime}=4 \int d z^{\prime} \int d r^{\prime} \frac{\rho\left(r^{\prime}, z^{\prime}\right) r^{\prime}}{\sqrt{\left(r+r^{\prime}\right)^{2}+\left(z-z^{\prime}\right)^{2}}} \mathcal{K}\left(\frac{4 r r^{\prime}}{\left(r+r^{\prime}\right)^{2}+\left(z-z^{\prime}\right)^{2}}\right)
$$


where $\mathcal{K}(x)$ is the complete elliptic integral of the first kind. More details about the calculation and numerical implementation of this integral is given in the Appendix.

After convergence of the iteration procedure, the total energy is given by

$$
E_{\text {exciton }}=\epsilon_{e}+\epsilon_{h}+\frac{e^{2}}{4 \pi \epsilon} \iint \frac{\rho_{e}(r, z) \rho_{h}\left(r^{\prime}, z^{\prime}\right)}{\left|\mathbf{r}-\mathbf{r}^{\prime}\right|} d \mathbf{r} d \mathbf{r}^{\prime}
$$

The contribution of the correlation to the total energy is neglected in HF, but for the self-assembled quantum dots, it is expected to be less than $2 \%$ [5] and for type II dots this will be even less.

\section{NUMERICAL RESULTS IN THE ABSENCE OF AN APPLIED MAGNETIC FIELD}

In our model system, there are two main parameters which can be varied: the radius of the disk $R$, and the thickness of the disk $d$. In the first part of our numerical study we will investigate the influence of these parameters on the exciton energy and wavefunction in the absence of a magnetic field. Following material parameters, typical for the InP/InGaP dot system, were taken: $m_{e}=0.077 m_{0}, m_{h}=0.60 m_{0}, \epsilon=12.61, V_{e}=250 \mathrm{meV}$ and $V_{h}=-50 \mathrm{meV}$.

The variation of the disk radius $R$ and thickness $d$ has large consequences for the hole wavefunction. Namely, the hole always tends to sit as closely as possible to the electron. In this way, when the disk thickness is larger than its diameter $(d>>2 R)$, the hole will prefer to sit at the radial boundary of the disk. When we consider very thin disks however $(d<<2 R)$, it will be much more favourable for the hole to be located above and below the disk. This state was not possible in our previous study [21] of the two-dimensional flat quantum dot, where the hole was forced to sit at the radial boundary of the dot. To illustrate this, we calculated the probability to find the hole at the radial boundary of the disk as

$$
P_{\text {side }}=2 \pi \int_{-\infty}^{\infty} d z_{h} \int_{R}^{\infty} d r_{h} r_{h}\left|\Psi_{h}\left(r_{h}, z_{h}\right)\right|^{2}
$$

When calculating this value for varying $R$ and $d$, we obtained a phase diagram for the position of the hole wavefunction, which is shown in Fig. 1(a). The hole confinement potential in all these calculations was fixed to $V_{h}=-50 \mathrm{meV}$. The solid curve indicates where $50 \%$ of the hole is located at the radial boundary, for the dashed curves it is respectively $25 \%$ and $75 \%$. In order to have a more visual picture of the hole state, we present contourplots of the hole wavefunction $\left|\Psi_{h}\left(r_{h}, z_{h}\right)\right|^{2}$ for three characteristic situations in the phase diagram, as indicated by the numbered stars. Fig. 1(b) shows the hole density for $R=4 n m, d=12 n m$, where the dotted lines indicate the boundary of the quantum disk. We clearly see that the hole is mainly situated at the radial side of the disk. Fig. 1(c) was made for $R=8 \mathrm{~nm}$, $d=8 \mathrm{~nm}$ and at this position, exactly $50 \%$ of the wavefunction is located at $r_{h}>R$. The third plot, Fig. 1(d), depicts the result for a thin disk, with $R=12 \mathrm{~nm}$ and $d=4 \mathrm{~nm}$, where the hole wavefunction is mostly located above and below the disk, where it is nearer to the electron.

Thus we can distinguish between two main systems: a) a disk-like system with $d<<2 R$, where the hole will be located above or below the quantum disk, and b) a pillar-like system with $d>>2 R$, where the hole sits mainly at the radial boundary of the disk. The solid line in Fig. 1(a) can be approximated by the curve $d=1.76 R-5.41 \mathrm{~nm}$. We will see in the following section that a magnetic field has a very different effect on these two classes of systems.

\section{RESULTS FOR A PERPENDICULAR APPLIED MAGNETIC FIELD}

\section{A. Single dot: a disk-like system}

After having discussed the influence of the main parameters of our system, we are now ready to consider an extra feature, i.e. a magnetic field applied along the $z$-direction, parallel to the growth direction. The magnetic field will squeeze the wavefunctions in the radial direction. When $d<<2 R$, the hole is above and below the disk, and will be pressed stronger to the center of the disk. Fig. 2 depicts the exciton energy as a function of the magnetic field for a disk with $R=10 \mathrm{~nm}$ and $d=2 \mathrm{~nm}$ for two different values of the hole angular momentum $l_{h}$. Note that this exciton energy includes both the one particle energies and the Coulomb energy, i.e. $E_{\text {exciton }}=E_{e}+E_{h}-E_{\text {coul }}$. In the present case the single hole energy $E_{h}$ will be 0 , as the single hole is not confined. The single electron energies at $B=0 T$ and $B=50 T$ are given by respectively $E_{e}=192.6 \mathrm{meV}$ and $E_{e}=212.3 \mathrm{meV}$. We find a strong enhancement of the exciton energy of almost $20 \mathrm{meV}$ for $B=0 T \rightarrow B=50 T$. Due to the magnetic field, the electron and hole are both pushed closer to the center, which enhances the Coulomb interaction. However, also the one particle electron energy 
increases drastically with increasing magnetic field, thus cancelling the effect of the enhanced (negative) Coulomb energy. The two insets show what happens with the electron (dashed curves) and hole (full curves) wavefunctions when a magnetic field is applied. For $B=0 T$ (Fig. 2(a)) the electron is strongly confined in the quantum disk, and the hole is localized above and below the disk. From the figure, it is obvious that this position is energetically most favourable for the hole, since the hole will be very close to the electron. Because of the very small thickness, there is some smearing of the electron wavefunction out of the disk in the $z$-direction, which attracts the hole to this position. The corresponding wavefunctions for $B=50 T$ are plotted in Fig. 2(b), where again the dashed curve indicates the electron wavefunction, and the solid curve the hole. We see that both particles are compressed in the radial direction. For the electron, this leads to a further penetration of the wavefunction into the barrier. Because of this, the hole will be even stronger attracted to the top and bottom of the disk, which leads to a larger penetration of the hole in the disk. As a result, the hole wavefunction will also be slightly squeezed in the $z$-direction.

\section{B. Single dot: a pillar-like system}

The exciton energy as a function of the magnetic field is plotted in Fig. 3 for the case of $R=4 n m, d=12 n m$ and $V_{h}=-50 \mathrm{meV}$. An interesting feature which appears is the occurrence of (hole) angular momentum transitions with increasing magnetic field. The origin of the angular momentum transitions is the following. As discussed in Sec. III, the preferred position of the hole for a disk with a thickness larger than its diameter will be at the radial boundary of the disk. When a magnetic field is applied along the $z$-direction, the hole will be pushed against the border of the disk. Since the disk forms a barrier for the hole, it will be energetically more favourable to jump to a higher angular momentum $l_{h}$ state. Notice that $l_{h}$ is an approximate quantum number. This feature was also apparent in our previous study of planar type II dots [21], where we forced the hole to sit at the radial boundary. This is similar to an exciton in a quantum-ring structure [26,27. In the present study however, we allow for the hole to relax also in the $z$-direction. Therefore, for a small thickness of the disk (disk-like system), it will be much more favourable for the hole to move in the $z$-direction and only for thick enough disks, such $l_{h}$ transitions occur. Contourplots of the wavefunctions are shown, respectively for the electron at $B=0 T$ (inset of Fig. 3) and for the hole at $B=0 T$, $B=25 T, B=50 T$ and $B=90 T$ (Figs. 4(a)-(d)). We find that the electron is located in the center of the disk, with almost no penetration into the barrier. At $B=0 T$, the hole is mainly sitting at the radial boundary of the disk, although there is some extent of the wavefunction towards the top and bottom of the disk, and there is also some penetration into the dot. When increasing the magnetic field, the hole jumps to the $l_{h}=1$ state around $B \simeq 15 T$. We plotted the hole wavefunction at $B=25 T$ (Fig. 4(b)), and we see that the hole is pushed at the side, where it has now more space to extend in the radial direction. Further increasing the magnetic field, leads to a jump to $l_{h}=2$ at $B \simeq 45 T$ and to $l_{h}=3$ at $B \simeq 79 T$. The contourplots were made for respectively $B=50 T$ (Fig. 4(c)) and $B=90 T$ (Fig. 4(d)), and we see again that the hole is sitting at the side of the disk. Notice that, for all four plots, the probability to find the hole at $r \simeq 12 \mathrm{~nm}$ is almost the same, i.e. $\left|\Psi_{h}\left(r_{h}=12 n m, z_{h}=0\right)\right|^{2} \simeq 0.025$. In the absence of those $l_{h}$ transitions, the magnetic field would have compressed the hole much more strongly to the radial boundary of the disk which leads to a larger energy as is shown clearly by the solid curve in Fig. 3 .

In order to investigate the influence of the hole confinement potential $V_{h}$ on the angular momentum transitions, we made a phase diagram of the angular momentum state of the hole as a function of both the confinement potential and the magnetic field. This is shown in Fig. 5 for a disk with radius $R=6 \mathrm{~nm}$ and thickness $d=14 \mathrm{~nm}$. The figure shows that up to $V_{h}=-13 \mathrm{meV}$ no angular momentum transitions occur. In this region $V_{h}$ is too small to form a barrier for the hole, and the hole jumps inside the disk due to the Coulomb interaction, forming a type I system. We also see that the transition between type I and type II is very sharp. In our previous study of the planar dots [21], we found from a similar figure a re-entrant behaviour to the $l_{h}=0$ state for sufficient large magnetic fields. This is not so evident in the present case, as we find that the line which separates the type I from the type II behaviour is almost vertical.

From an experimental point of view, it is interesting to look at the probability for recombination of the exciton, which is proportional to the square of the overlap integral

$$
I=\int \Psi_{e}(\mathbf{r}) \Psi_{h}(\mathbf{r}) d \mathbf{r}=\int_{0}^{2 \pi} e^{i\left(l_{e}+l_{h}\right) \varphi} d \varphi \int_{0}^{\infty} \psi_{e}(r) \psi_{h}(r) r d r .
$$

The integral over the angle gives $2 \pi \delta_{l_{e}+l_{h}}$. This means that the probability for de-excitation is only non-zero for $l_{e}+l_{h}=0$. This implies that, after an angular momentum transition, the probability for recombination decreases strongly, leading to a vanishing of the photoluminescence (PL) spectrum after a certain value of the magnetic field. Fig. 6 depicts the overlap integral $I$ as a function of the confinement potential of the hole, for both $B=0 T$ (solid curve) and $B=25 T$ (dashed curve). For $B=0 T$ the ground state will always be the $l_{h}=0$ state, and the overlap 
integral will always be non-zero. However, as the system goes from type I to type II we see a strong decrease of the overlap, because the exciton becomes spatially indirect. For $B=25 T$ we find a different picture: after the system has become type II, the overlap integral becomes zero. This is due to the fact that at $B=25 T$ an angular momentum transition has occurred and the condition $l_{e}+l_{h}=0$ is no longer fulfilled.

\section{Two vertically coupled dots}

The inclusion of the $z$-direction in our calculation enables us to investigate systems of vertically coupled quantum dots. We have now an extra parameter to vary, namely the interdot distance $d_{z}$. In this first part, we focus on two stacked dots of the same size, namely radius $R=6 \mathrm{~nm}$ and thickness $d=6 \mathrm{~nm}$, and with $d_{z}=3.6 \mathrm{~nm}$. The total stack height of $15.6 \mathrm{~nm}$ is thus larger than the diameter of the disks, being $12 \mathrm{~nm}$. Therefore we expect to find a magnetic field behaviour which resembles that of the single disk pillar-like system. The result for the exciton energy as function of the magnetic field is shown in Fig. 7. Indeed we find angular momentum transitions with increasing magnetic field. The inset, Fig. 7(a), shows a contourplot of the electron wavefunction at $B=0 T$, which is symmetrically distributed over the two disks. The Coulomb interaction attracts the hole as close as possible to the electron, in this case the radial boundary of the disks. As one can see in Fig. 7(b), the hole tends also to sit between the disks, to be even closer to the electron. A magnetic field acts on the wavefunctions in the radial direction, and thereby pushes the hole stronger between the disks. There is however not enough space, and therefore it will be energetically more favourable for the hole to jump to a higher $l_{h}$-state.

When giving a closer look at the behaviour of the different $l_{h}$-states in a magnetic field (Fig. 7), a remarkable feature appears: the $l_{h}=0$ curve exhibits a kind of kink around $B=15 T$, and we find a similar feature for $l_{h}=1$ around $B=45 T$. Investigation of the wavefunctions (Figs. 8(a-c)) learns us that we are dealing with a spontaneous symmetry breaking [28]. The corresponding hole wavefunction at $B=15 T, 30 T$ and $50 T$, for $l_{h}=0$ is shown in Fig. 8(a,b,c) respectively, where one can see clearly the increase of asymmetry with magnetic field. But for such magnetic fields the $l_{h}=0$ state is not the ground state. This means that the jump to a higher angular momentum state is still preferred above the asymmetric state (see Fig. $8(\mathrm{~d})$ for $l_{h}=3$ and $B=50 T$ ). We also found that with increasing $l_{h}$ the symmetry breaking will occur at larger magnetic fields.

In order to investigate whether or not the ground state configuration is asymmetric, we calculated

$$
\Delta P_{\text {asymm }}=2 \pi \int_{0}^{\infty} d r_{h} r_{h}\left(\int_{0}^{\infty} d z_{h}-\int_{-\infty}^{0} d z_{h}\right)\left|\Psi_{h}\left(r_{h}, z_{h}\right)\right|^{2},
$$

which expresses the degree of asymmetry of the hole wavefunction in the $z$-direction. This quantity is plotted in Fig. 9 as function of the magnetic field for $l_{h}=0,1,2,3$. This plot confirms that after $B \simeq 15 T$ the $l_{h}=0$ state becomes highly asymmetric, and for higher $B$ also $l_{h}=1$ becomes asymmetric. The asymmetric states for the subsequent $l_{h}$ states occur for $B>50 T$. The contourplot of the hole wavefunction at $B=50 T$ is shown in Fig. 8(d) for the ground state, which is $l_{h}=3$. The inset of Fig. 9 shows the dipole moment for the different $l_{h}$ states with increasing magnetic field. This is an interesting quantity, as it can be measured experimentally. Note that for our symmetric system, we have the unique feature that a magnetic field is able to induce a dipole moment!

We can understand the spontaneous symmetry breaking as follows. In the asymmetric case the electron wavefunction is of course attracted to the hole (see Fig. 8(b), dashed lines) and thus stronger confined, in this way increasing its single particle energy. Therefore, the broken symmetry state can only occur if it can overcome this increase in energy due to a larger Coulomb attraction between electron and hole. We mentioned that an increasing magnetic field favoured the broken symmetry state. The reason is that with increasing magnetic field the electron becomes stronger confined in the dot, and consequently expells more in the $z$-direction. In the broken symmetry case it even expells more, resulting in a larger overlap with the hole, which can eventually make the asymmetric state lower in energy than the symmetric state. If this is true, than the broken symmetry would occur even sooner with increasing magnetic field for thinner disks, where the electron is expelled more in the barrier material in the $z$-direction. That this is indeed the case is shown in Fig. 10 where for two thin vertically coupled disks $\left(R=12 \mathrm{~nm}, d=3 \mathrm{~nm}\right.$ and $d_{z}=3 \mathrm{~nm}$, $l_{h}=0$ ) we find already a broken symmetry state for zero magnetic field. In this case, this broken symmetry state is also the ground state.

\section{Two vertically coupled truncated cones}

It is now interesting to check whether the above angular momentum transitions survive in a system which is completely asymmetric from the beginning, such as a system of two vertically coupled truncated cones. The two 
cones are equal, and defined by a base radius $R_{b}$ of $7 \mathrm{~nm}$, a thickness $d=6 \mathrm{~nm}$, and an angle of $71^{\circ}$ (dashed lines in the insets of Fig. 11). The hole and electron wavefunctions (Figs. 11 (a) and (b)) show indeed the asymmetric behaviour, at $B=0 T$ and for $l_{h}=0$. The result for the exciton energy as a function of the magnetic field is depicted in Fig. 11. The interesting feature is that, even for this system, angular momentum transitions occur. Since there is still some part of the hole wavefunction located at the radial border and also some part between the disks, it appears to be still more favourable for the hole to jump to the higher $l_{h}$-state than to move more and more below the disk. Figs. 12(a-c) show contourplots of the hole wavefunction in the ground state for subsequent $l_{h}$-states, respectively $l_{h}=1$ at $B=20 T, l_{h}=2$ at $B=30 T$, and $l_{h}=3$ at $B=50 T$.

\section{E. Three vertically coupled dots: small interdot distance $d_{z}$}

For simplicity we consider only the case of three identical dots. The first system under investigation contains three vertically coupled quantum disks, each with disk radius $R=8 \mathrm{~nm}$, disk thickness $d=3 \mathrm{~nm}$, and with interdot distance $d_{z}=3 \mathrm{~nm}$. In this case the confinement of the hole $V_{h}$ was taken to be $-30 \mathrm{meV}$. Fig. 13 shows the result for the exciton energy as a function of the magnetic field. Similar to the case of the pillar-like single dot, we find angular momentum transitions with increasing magnetic field. The origin of these angular momentum transitions can be understood by looking at the wavefunctions. The insets of Fig. 13 show the electron wavefunctions at (a) $B=0 T$ and (b) $B=50 T$, and it appears that the main part is located in the middle dot, whereas there is some small extent of the wavefunction into both the upper and lower dots. At $B=50 T$, the wavefunction is more squeezed in the radial direction. The evolution of the hole wavefunction with increasing magnetic field is depicted in Fig. 14. At $B=0 T$ (Fig. 14(a)), the main part of the hole wavefunction is situated at the radial boundary of the stacked system and its probability to sit at the radial side is $P_{\text {side }}=73 \%$. There is some extent of the wavefunction towards the top and bottom of the stack, and also between the dots. When the magnetic field increases (Fig. 14(b), $B=10 T$ ), the wavefunction is pushed further between the disks, because it prefers to sit as closely as possible to the electron. However, there is not enough space between the disks to confine the whole wavefunction, and therefore it is energetically more favourable for the hole to jump to a higher angular momentum state. Further increasing the magnetic field leads to more angular momentum transitions (Figs. 14(c-f)).

As can be seen from the wavefunctions, the ground state for this system is always symmetric. The asymmetry, as found in the two-disks system, is however still present, but it turns out not to be the ground state. For the $l_{h}=0$ state, the wavefunctions become asymmetric at about $B=20 T$,resulting in a slight bow in the full curve of Fig. 13. The fact that the asymmetry is less pronounced in this system can be understood as follows: in the three-disks system the main part of the electron is located in the middle disk. This is in contrast to the two-disks system, where the electron wavefunction has equal parts sitting in the two disks. Even when an asymmetry is induced in the present three-disks system, the electron will still be mainly located in the middle disk, and therefore the hole will sit more tightly at the radial boundary than was the case for the two coupled disks.

\section{F. Three vertically coupled dots: large interdot distance $d_{z}$}

When we increase the interdot distance $d_{z}$ up to $5.5 \mathrm{~nm}$, the hole will start to be confined between the disks. Fig. 15 shows that there are no angular momentum transitions for this system and it resembles very much the narrow disk-like system (see Fig. 2). In this figure the solid curve denotes the $l_{h}=0$ state, whereas the dashed curve denotes the $l_{h}=1$ curve. We turn again to the wavefunctions to explain this behaviour. The electron is sitting mainly in the middle dot, this time with smaller extent into the upper and lower dots (Figs. 15(a) and (b) for respectively $B=0 T$ and $B=50 T$ ). The contourplot for the hole wavefunction (Fig. 16(a)) shows that already at $B=0 T$ the hole is completely situated between the disks. This is the preferred place for the hole, as it tends to sit as close as possible to the electron. Further increasing the magnetic field, squeezes the wavefunction more in the radial direction (Fig. 16(b)). As there is still enough space between the disks, there is no need for the hole to jump to a higher angular momentum state, and $l_{h}=0$ remains the ground state over the whole $B$-region.

For this case, no asymmetry occurs up to $B=50 T$. This means that this system is quite stable against spontaneous symmetry breaking. This stability is due to the fact that again the electron is strongly located in the middle disk, and that the hole is now sitting between the disks instead at the radial boundary. 


\section{G. Revision of the results for the single disk systems}

We now turn back to the single disk systems, both the disk-like and the pillar-like, where we did not talk about broken symmetry solutions. However also in this case they can be found.

We find that the disk-like system, i.e. $R=10 \mathrm{~nm}$ and $d=2 \mathrm{~nm}$, is perfectly stable up to $B=50 T$ and we find no asymmetric behaviour. This is in perfect agreement with the result for the three coupled disks with $d_{z}=5.5 \mathrm{~nm}$. Indeed, for the disk-like system we have again the electron sitting in the disk with the hole above and below the disks, which is quite similar to the second three-disks system.

For the pillar-like system, i.e. $R=4 \mathrm{~nm}$ and $d=12 \mathrm{~nm}$, we do find an asymmetry, but only for the $l_{h}=0$ state and at very high magnetic fields, i.e. starting around $B \simeq 80 T$. The difference in energy between the states with symmetric and antisymmetric wavefunctions is only $0.08 \mathrm{meV}$ for $B=90 \mathrm{~T}$. The ground state however remains symmetric over the total considered $B$-region. Thus the wavefunctions, as plotted in Fig. 4, remain unchanged.

\section{CONCLUSIONS}

We studied an exciton in a type II quantum disk of radius $R$ and thickness $d$. For the confinement potential we took a hard wall of finite height, with the hole located in the barrier, which is only confined by the interaction with the electron, confined inside the dot. We calculated the exciton energy and wavefunction using the Hartree-Fock mesh method, which allows us to start without any knowledge of the single hole wavefunction. We limit ourselves to the study of a model system in which strain effects are neglected.

In the first part, we examined the case of an exciton in a single disk. First we investigated the exciton properties in the absence of a magnetic field. The calculation of the probability for the hole wavefunction to sit at the radial boundary tells us that we can distinguish two "regimes": disk-like systems with $d<<2 R$, where the hole will prefer to sit above and below the quantum disk, and pillar-like systems with $d>>2 R$, where the hole will be located at the radial boundary of the disk.

Applying a magnetic field along the $z$-direction results in a different behaviour for these two systems. For the disk-like system, the hole is squeezed in the radial direction by the magnetic field, and the ground state of the system is the $l_{h}=0$ state for all values of $B$. For the pillar-like system, the magnetic field pushes the hole closer to the disk boundary, which forms a barrier for the hole. Therefore it will be energetically more favourable for the hole to jump to a higher angular momentum $l_{h}$ state, as then the wavefunction is able to relax away from the radial boundary. With increasing magnetic field, we find successive $l_{h}$ transitions.

The investigation of a system of two vertically coupled disks was done in order to compare its magnetic field dependence with the pillar-like single-disk system. As was expected, we find again angular momentum transitions of the ground state. Moreover, a new feature appeared in this study, namely a spontaneous symmetry breaking, induced by both the Coulomb interaction and the magnetic field. We found though that with increasing magnetic field, it is still preferable for the hole to jump to a higher angular momentum state. Investigation of a system of two vertically coupled truncated cones learned us that also in this case angular momentum transitions occur, although the system is highly asymmetric.

A system of three vertically coupled disks was investigated in the third part of the paper. It was shown that the system with small interdot distance $d_{z}$ behaves similarly to that of the single pillar-like disk. Again we found angular momentum transitions with increasing magnetic field. For a larger interdot distance however, the hole wavefunction tends to sit between the disks. In this case, an increasing magnetic field does not lead to angular momentum transitions anymore, and the $l_{h}=0$ state remains the ground state. No spontaneous symmetry broken states are found as the ground state. This is similar to the single disk system where symmetry broken states occur only as excited states.

\section{ACKNOWLEDGMENTS}

K. L. J. is supported by the "Instituut voor de aanmoediging van Innovatie door Wetenschap en Technologie in Vlaanderen" (IWT-Vl) and B. P. is a post-doctoral researcher with the Flemish Science Foundation (FWO-Vl.). Discussions with M. Hayne, M. Tădić and A. Matulis are gratefully acknowledged. Part of this work was supported by the FWO-Vl, The Belgian Interuniversity Attraction Poles (IUAP), the Flemish Concerted Action (GOA) Programmes, the University of Antwerp (VIS) and European Commission GROWTH programme NANOMAT project, contract no. GSRD-CT-2001-00545. 


\section{APPENDIX: CALCULATION OF THE HARTREE INTEGRAL}

The Hartree integral expresses the interaction effect of one particle on another, and is given by

$$
\int \frac{\rho\left(r^{\prime}, z^{\prime}\right)}{\left|\mathbf{r}-\mathbf{r}^{\prime}\right|} d \mathbf{r}^{\prime}=\int d z^{\prime} \int d r^{\prime} r^{\prime} \int d \varphi^{\prime} \frac{\rho\left(r^{\prime}, z^{\prime}\right)}{\sqrt{r^{2}+r^{\prime 2}-2 r r^{\prime} \cos \left(\varphi-\varphi^{\prime}\right)+\left(z-z^{\prime}\right)^{2}}}
$$

which contains a $z$-dependence which was absent in our previous work (see Appendix of Ref. 21]). We can remove the $\varphi$-dependence, since we deal with a cylindrical symmetric system. The integral over the angle becomes the complete elliptic integral of the first kind, which converts Eq. (A1) into

$$
4 \int d z^{\prime} \int \frac{\rho\left(r^{\prime}, z^{\prime}\right) r^{\prime}}{\sqrt{\left(r+r^{\prime}\right)^{2}+\left(z-z^{\prime}\right)^{2}}} \mathcal{K}\left(\frac{4 r r^{\prime}}{\left(r+r^{\prime}\right)^{2}+\left(z-z^{\prime}\right)^{2}}\right) d r^{\prime} .
$$

The radial integral has to be solved numerically. We use a polynomial approximation for the elliptic function [29], namely

$$
\mathcal{K}(x)=\left[a_{0}+a_{1} x^{\prime}+a_{2} x^{\prime 2}\right]-\left[b_{0}+b_{1} x^{\prime}+b_{2} x^{\prime 2}\right] \ln \left(x^{\prime}\right),
$$

with $x^{\prime}=1-x$ and where the coefficients $a_{i}$ and $b_{i}$ are given in Ref. [29]. The commonly used trapezoidal rule will give bad results, as this elliptic function implies the appearance of a logaritmic divergence in the integrand. Therefore we introduced the so-called 'logaritmically weighted method' which takes care of this problem.

Generally, the following integral can be considered:

$$
I(r, z)=\int_{0}^{1} d x F(x) \ln \left(\frac{(x-r)^{2}+\left(z-z^{\prime}\right)^{2}}{(x+r)^{2}+\left(z-z^{\prime}\right)^{2}}\right),
$$

which, after transformation, becomes

$$
I(r, z)=\sum_{i=0}^{N-1} \int_{0}^{h} d x F(x+h i) \ln \left(\frac{(x-(r-h i))^{2}+\left(z-z^{\prime}\right)^{2}}{(x+(r+h i))^{2}+\left(z-z^{\prime}\right)^{2}}\right),
$$

with $h$ the discretization step and $N$ the number of steps. If we replace $F(x+h i)$ by $F_{i}+\left[F_{i+1}-F_{i}\right](x / h)$, we can write (A5) as

$$
I(r, z)=\sum_{i=0}^{N-1}\left\{F_{i} A_{i}(r, z)+\left[F_{i+1}-F_{i}\right] C_{i}(r, z)\right\},
$$

and the remaining problem is the calculation of the coefficients $A_{i}(r, z)$ and $C_{i}(r, z)$. The integrals which determine the coefficients can be solved exactly, which leads to the following results:

$$
A_{i}(r, z)=a(h-(r-h i))-a(h+(r+h i)),
$$

with

$$
\begin{aligned}
& a(y)=P(y)+P(h-y)+2\left|z-z^{\prime}\right|\left(\arctan \frac{y}{\left|z-z^{\prime}\right|}+\arctan \frac{h-y}{\left|z-z^{\prime}\right|}\right), \\
& P(y)=y \ln \left(y^{2}+\left(z-z^{\prime}\right)^{2}\right),
\end{aligned}
$$

and

$$
C_{i}(r, z)=h^{-1}[c(h-(r-h i))-c(h+(r+h i))]-2 r,
$$

with 


$$
\begin{aligned}
c(y)= & \frac{1}{2}\left(y(2 h-y)^{2}+\left(z-z^{\prime}\right)^{2}\right) Q(y)+\frac{1}{2}\left((h-y)^{2}-\left(z-z^{\prime}\right)^{2}\right) Q(h-y) \\
& +2\left|z-z^{\prime}\right|(h-y)\left(\arctan \frac{y}{\left|z-z^{\prime}\right|}+\arctan \frac{h-y}{\left|z-z^{\prime}\right|}\right) \\
Q(y)= & \ln \left(y^{2}+\left(z-z^{\prime}\right)^{2}\right) .
\end{aligned}
$$

* $\quad$ Electronic mail: karenj@uia.ua.ac.be

- Electronic mail: bpartoen@uia.ua.ac.be

$\dagger \quad$ Electronic mail: peeters@uia.ua.ac.be

[1] For a review, see e.g., D. Bimberg, M. Grundmann, and N.N Ledentsov, in Quantum Dot Heterostructures (John Wiley \& Sons, Chichester, 1999).

[2] A. Polimeni, S.T. Stoddart, M. Henini, L. Eaves, P.C. Main, K. Uchida, R.K. Hayden, and N. Miura, Physica E 2, 662 (1998).

[3] L.R. Wilson, D.J. Mowbray, M.S. Skolnick, M. Morifuji, M.J. Steer, I.A. Larkin, and M. Hopkinson, Phys. Rev. B 57, R2073 (1998).

[4] O. Stier, M. Grundmann, and D. Bimberg, Phys. Rev. B 59, 5688 (1999).

[5] M. Brasken, M. Lindberg, D. Sundholm, and J. Olsen, Phys. Rev. B 61, 7652 (2000).

[6] M. Bayer, A. Schmidt, A Forchel, F. Faller, T.L. Reinecke, P.A. Knipp, A.A. Dremin, and V.D. Kulakovskii, Phys. Rev. Lett. 74, 3439 (1995).

[7] J. Song and S.E. Ulloa, Phys. Rev. B 52, 9015 (1995).

[8] K.L. Janssens, F.M. Peeters, and V.A. Schweigert, Phys. Rev. B 63, 205311 (2001).

[9] M. Korkusinski and P. Hawrylak, Phys. Rev. B 63, 195311 (2001).

[10] L. Müller-Kirsch, R. Heitz, A. Schliwa, O. stier, and D. Bimberg, Appl. Phys. Lett. 78, 1418 (2001).

[11] R. Heitz, N.N. Ledentsov, D. Bimberg, M.V. Maximov, A.Yu. Egorov, V.M. Ustinov, A.E. Zhukov, Zh.I. Alferov, G.E. Cirlin, I.P. Shoshnikov, N.D. Zakharov, P. Werner, and U. Gösele, Appl. Phys. Lett. 74, 1701 (1999).

[12] C.-K. Sun, G. Wang, J.E. Bowers, B. Brar, H.-R. Blank, H. Kroemer, and M. Pilkuhn, Appl. Phys. Lett. 68, 1543 (1996).

[13] S. Nomura, L. Samuelson, M.-E. Pistol, K. Uchida, N. Miura, T. Sugano, and Y. Aoyagi, Appl. Phys. Lett. 71, 2316 (1997).

[14] M. Hayne, R. Provoost, M.K. Zundel, Y.M. Manz, K. Eberl, and V.V. Moshchalkov, Phys. Rev. B 62, 10324 (2000).

[15] M. Sugisaki, H.-W. Ren, K. Nishi, S. Sugou, T. Okuno, and Y. Masumoto, Physica B 256-258, 169 (1998).

[16] L. Müller-Kirsch, R. Heitz, A. Schliwa, O. Stier, D. Bimberg, H, Kirmse, and W. Neumann, Appl. Phys. Lett. 78, 1418 (2001).

[17] F. Hatami, M. Grundmann, N.N. Ledentsov, F. Heinrichsdorff, R. Heitz, J. Böhrer, D. Bimberg, S.S. Ruvimov, P. Werner, V.M. Ustinov, P.S. Kop'ev, and Zh.I. Alferov, Phys. Rev B 57, 4635 (1998).

[18] C. Pryor, M.-E. Pistol, and L. Samuelson, Phys. Rev B 56, 10404 (1997).

[19] M. Tadic, F.M. Peeters, and K.L. Janssens, submitted to Phys. Rev. B.

[20] S. Nomura, L. Samuelson, C. Pryor, M.-E. Pistol, M. Stopa, K. Uchida, N. Miura, T. Sugano, and Y. Aoyagi, Phys. Rev. B 58, 6744 (1998).

[21] K.L. Janssens, B. Partoens, and F.M. Peeters, Phys. Rev. B 64, 155324 (2001).

[22] Ph. Lelong, K. Suzuki, G. Bastard, H. Sakaki, and Y. Arakawa, Physica E 7, 393 (2000).

[23] A.B. Kalameitsev, V.M. Kovalev, and A.O. Govorov, JETP Lett. 68, 669 (1998).

[24] M. V. Ivanov, J. Phys. B: At. Mol. Opt. Phys. 27, 4513 (1994); ibid., Phys. Rev. A 61, 22505 (2000).

[25] F.M. Peeters and V.A. Schweigert, Phys. Rev. B 53, 1468 (1996).

[26] S.E. Ulloa, A.O. Govorov, A.V. Kalameitsev, R. Warburton, and K. Karrai, cond-mat/0111006.

[27] A.O. Govorov, A.V. Kalameitsev, R. Warburton, K. Karrai, and S.E. Ulloa, cond-mat/0111007.

[28] We allowed for broken symmetry solutions by manually pushing the electron single particle wavefunction $0.1 \%$ more to the positive $z$-direction with respect to the negative $z$-direction in the first iteration step.

[29] M. Abramowitz and I. A. Stegun, Handbook of Mathematical Functions (National Bureau of Standards, Washington DC, 1970), expression 17.3.34. 
FIG. 1. (a) Phase diagram of the probability for the hole wavefunction to sit at the radial border of the disk, as a function of both $R$ and $d$. (b), (c) and (d) are contourplots of the hole wavefunction, at the positions as indicated by the corresponding numbers on the main figure. The dashed lines in the contourplots indicate the boundary of the disk.

FIG. 2. The variation of the exciton energy with increasing magnetic field, for a disk with thickness $d=2 \mathrm{~nm}$ and radius $R=10 \mathrm{~nm}$ and two values of the hole angular momentum. The insets show the electron (dashed curve) and hole (solid curve) wavefunction for (a) $B=0 T$ and (b) $B=50 T$.

FIG. 3. Evolution of the exciton energy with increasing magnetic field, for a disk with thickness $d=12 \mathrm{~nm}$ and radius $R=4 \mathrm{~nm}$. Increasing the magnetic field, leads to successive transitions of the hole angular momentum $l_{h}$ indicated by the arrows. The inset depicts a countourplot of the electron wavefunction at $B=0 T$. The dashed line indicates the disk boundary.

FIG. 4. Contourplots of the hole wavefunction for a disk with $R=4 n m$ and $d=12 n m$, at (a) $B=0 T$, (b) $B=25 T$, (c) $B=50 T$ and (d) $B=90 T$. The dashed lines indicate the disk boundary.

FIG. 5. Phase diagram of the angular momentum transitions as a function of the hole confinement potential $V_{h}$ and the magnetic field $B$, for a disk radius $R=6 \mathrm{~nm}$ and thickness $d=14 \mathrm{~nm}$.

FIG. 6. Overlap integral as a function of the confinement potential of the hole, for $B=0 T$ (solid curve) and $B=25 T$ (dashed curve).

FIG. 7. Exciton energy as function of the magnetic field, for two coupled disks with $R=6 \mathrm{~nm}, d=6 \mathrm{~nm}$ and $d_{z}=3.6 \mathrm{~nm}$. The insets show contourplots of the wavefunctions at $B=0 T$, for respectively the electron (a) and the hole (b).

FIG. 8. Contourplots of the hole wavefunction for two coupled disks, for $l_{h}=0$ at respectively $B=15 T$ (a), $B=30 T$ (b) and $B=50 T$ (c) and for $l_{h}=3$ at $B=50 T(\mathrm{~d})$.

FIG. 9. Degree of asymmetry of the different $l_{h}$ states as a function of the magnetic field. The inset shows the dipole moment as a function of the magnetic field.

FIG. 10. Contourplots of the electron (a) and hole (b) wavefunction at $B=0 T$ for two coupled disks with $R=12 n m$, $d=3 \mathrm{~nm}$ and $d_{z}=3 \mathrm{~nm}$.

FIG. 11. Evolution of the exciton energy with the magnetic field for two coupled truncated cones. The insets show contourplots of the electron (a) and hole (b) wavefunctions at $B=0 T$. The dashed lines indicate the disk boundary.

FIG. 12. Contourplots of the hole wavefunction at (a) $B=20 T$, (b) $B=30 T$ and (c) $B=50 T$ for a double dot system made out of truncated cones.

FIG. 13. Exciton energy as a function of the magnetic field, for three vertically coupled disks with interdot distance $d_{z}=3 n m$. Increasing the magnetic field leads to angular momentum transitions, which are indicated by the arrows. The insets show contourplots of the electron wavefunctions at respectively (a) $B=0 T$ and (b) $B=50 T$. The dashed lines indicate the disk boundaries.

FIG. 14. Contourplots of the hole wavefunction for (a) $B=0 T$, (b) $B=10 T$, (c) $B=15 T$, (d) $B=25 T$, (e) $B=35 T$ and (f) $B=50 T$. The dashed lines indicate the disk boundaries. 
FIG. 15. Exciton energy as a function of the magnetic field, for three vertically coupled disks with interdot distance $d_{z}=5.5 \mathrm{~nm}$. The insets are contourplots of the electron wavefunctions for (a) $B=0 T$ and (b) $B=50 T$. The dashed lines indicate the disk boundaries.

FIG. 16. Contourplots of the hole wavefunctions for (a) $B=0 T$ and (b) $B=50 T$. The dashed lines indicate the disk boundary. 
This figure "fig1.PNG" is available in "PNG" format from: http://arxiv.org/ps/cond-mat/0201026v1 
This figure "Fig2.PNG" is available in "PNG" format from: http://arxiv.org/ps/cond-mat/0201026v1 
This figure "Fig3.PNG" is available in "PNG" format from: http://arxiv.org/ps/cond-mat/0201026v1 
This figure "Fig4.PNG" is available in "PNG" format from: http://arxiv.org/ps/cond-mat/0201026v1 
This figure "Fig5.PNG" is available in "PNG" format from: http://arxiv.org/ps/cond-mat/0201026v1 
This figure "fig6.PNG" is available in "PNG" format from: http://arxiv.org/ps/cond-mat/0201026v1 
This figure "Fig7.PNG" is available in "PNG" format from: http://arxiv.org/ps/cond-mat/0201026v1 
This figure "Fig8.PNG" is available in "PNG" format from: http://arxiv.org/ps/cond-mat/0201026v1 
This figure "Fig9.PNG" is available in "PNG" format from: http://arxiv.org/ps/cond-mat/0201026v1 
This figure "fig10.PNG" is available in "PNG" format from: http://arxiv.org/ps/cond-mat/0201026v1 
This figure "Fig11.PNG" is available in "PNG" format from: http://arxiv.org/ps/cond-mat/0201026v1 
This figure "Fig12.PNG" is available in "PNG" format from: http://arxiv.org/ps/cond-mat/0201026v1 
This figure "Fig13.PNG" is available in "PNG" format from: http://arxiv.org/ps/cond-mat/0201026v1 
This figure "Fig14.PNG" is available in "PNG" format from: http://arxiv.org/ps/cond-mat/0201026v1 
This figure "Fig15.PNG" is available in "PNG" format from: http://arxiv.org/ps/cond-mat/0201026v1 
This figure "Fig16.PNG" is available in "PNG" format from: http://arxiv.org/ps/cond-mat/0201026v1 